\title{
Sulfuric Acid ([3-(3-Silicapropyl)sulfanyl]propyl)ester as a Recyclable Catalyst for the Synthesis of 4,4'-(Arylmethylene)bis(1H-pyrazol-5-ols)
}

\author{
Shekoofeh TAYEBI ${ }^{1}$, Mojtaba BAGHERNEJAD ${ }^{1}$, Dariush SABERI ${ }^{2}$, Khodabakhsh NIKNAM $^{1,2,{ }^{*}}$ \\ ${ }^{1}$ Department of Chemistry, Islamic Azad University, Gachsaran Branch, Gachsaran, Iran \\ ${ }^{2}$ Department of Chemistry, Faculty of Sciences, Persian Gulf University, Bushehr 75169, Iran
}

\begin{abstract}
Sulfuric acid ([3-(3-silicapropyl)sulfanyl]propyl)ester is employed as a recyclable catalyst for the condensation reaction between aromatic aldehydes and 3-methyl-1-phenyl-5-pyrazolone. This condensation reaction was performed in ethanol under refluxing conditions giving 4,4-alkylmethylene-bis(3-methyl-5-pyrazolones) in 74-90\% yields. The heterogeneous catalyst was recycled and used in eleven runs for the reaction between benzaldehyde and 3-methyl-1-phenyl-5-pyrazolone without losing catalytic activity.
\end{abstract}

Key words: sulfuric acid ([3-(3-silicapropyl)sulfanyl]propyl)ester; silica-bonded $N$-propyl sulfamic acid; 3-methyl-1-phenyl-5-pyrazolone; aldehydes

CLC number: O643 Document code: A

Received 9 March 2011. Accepted 8 July 2011.

*Corresponding author.Tel: +98-771-4541494; Fax:+98-771-4545188; E-mail: niknam@pgu.ac.ir,khniknam@gmail.com

English edition available online at Elsevier ScienceDirect (http://www.sciencedirect.com/science/journal/18722067).

The development of heterogeneous catalysts for organic synthesis has become a major area of research. The potential advantages of these materials over homogeneous systems (simplified recovery, reusability, and the potential for incorporation in continuous reactors and microreactors) could lead to novel, environmentally benign chemical procedures for academia and industry [1,2]. The application of solid acids in organic transformations is important because they have many advantages including ease of handling, decreased reactor and plant corrosion problems, and their disposal is more environmentally friendly [3-21].

Several types of solid sulfonic acid functionalized silica (both amorphous and ordered) have been synthesized and applied as alternatives to traditional sulfonic acid resins and homogeneous acids for catalyzing chemical transformations [3-8]. In continuation of our studies into the design and application of solid acid catalysts in organic transformations
[7-21], we thus describe the application of sulfuric acid ([3-(3-silicapropyl)sulfanyl]propyl)ester (SASPSPE) as recyclable catalyst for the synthesis of $4,4^{\prime}$-alkylmethylenebis(3-methyl-5-pyrazolones) (Scheme 1).

Pyrazoles are an important class of bio-active drug targets in the pharmaceutical industry as they are the core structure of numerous biologically active compounds [22-24]. For example; they exhibit anti-anxiety, antipyretic, analgesic and anti-inflammatory properties. 2,4-Dihydro-3 $H$-pyrazol3-one derivatives including 4,4'-(arylmethylene)bis(3methyl-1-phenyl-1H-pyrazol-5-ols) have a broad spectrum of approved biological activity and are used as anti-inflammatory [25], antipyretic [26], gastric secretion stimulatory [27], antidepressant [28], antibacterial [29] and antifilarial agents [30]. Moreover, the corresponding 4,4'-(arylmethylene)bis(1H-pyrazol-5-ols) are applied as fungicides [31], pesticides [32], insecticides [33], and dye-

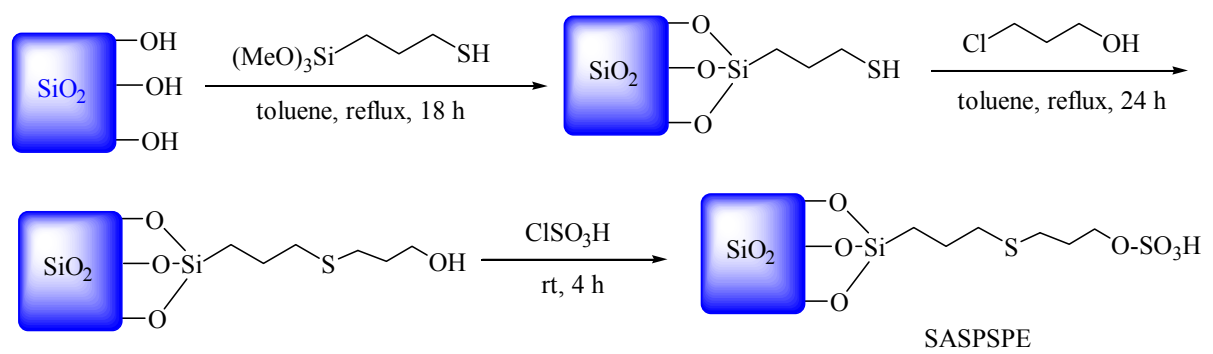

Scheme 1. Preparation of sulfuric acid ([3-(3-silicapropyl)sulfanyl]propyl)ester (SASPSPE). 
stuffs [34-36], and as chelating and extraction reagents for different metal ions [37,38]. The conventional chemical approach to 4,4'-(arylmethylene)bis(3-methyl-1-phenylpyrazol-5-ols) involves the successive Knoevenagel synthesis of the corresponding arylidenepyrazolones and its base-promoted Michael reaction as well as the one-pot tandem Knoevenagel-Michael reaction of arylaldehydes with two equivalents of 5-methyl-2-phenyl-2,4-dihydro-3 $\mathrm{H}$ pyrazol-3-one performed under various reaction conditions $[39,40]$. The first set of procedures is concerned with the catalysis of the components with piperidine in ethanolic solution [41,42]. The second set of methods involves the non-catalyzed tandem Knoevenagel-Michael reaction under neutral conditions in either ethanol [43] or benzene [44] solutions. Although the corresponding 4,4'-(arylmethylene) bis( $1 H$-pyrazol-5-ols) are obtained in reliable $70 \%-90 \%$ yields, the reaction requires $3-12 \mathrm{~h}$ of initial reflux and a further $24 \mathrm{~h}$ under ambient temperature to reach completion. Recently, sodium dodecyl sulfate [45], an electrocatalytic procedure [46], CAN [47], and silica-bonded $S$-sulfonic acid [17] were reported for the synthesis of 4,4'-(arylmethylene) bis(3-methyl-1-phenyl-1H-pyrazol-5-ols). However, most of these methods suffer from at least one limitation that may include moderate yields, long reaction times, harsh reaction conditions, or tedious workup procedures.

\section{Experimental}

\subsection{Catalyst preparation}

\subsubsection{3-(3-Silicapropylthio)-1-propanol}

3-Chloro-1-propanol (5 mmol, $0.473 \mathrm{~g}$ ) was added to a magnetically stirred mixture of 3-mercaptopropylsilica (10 $\mathrm{g})$ in toluene $(30 \mathrm{ml})$ and then a few drops of triethyl amine was added and the resulting mixture was refluxed for $24 \mathrm{~h}$. The mixture was filtered and the solid was washed with ethanol $(20 \mathrm{ml} \times 3)$ and then dried in an oven. 3-(3-Silicapropylthio)-1-propanol was obtained as a cream powder (10.3 g).

\subsubsection{SASPSPE}

To a mixture of 3-(3-silicapropylthio)-1-propanol (5 g) in chloroform $(20 \mathrm{ml})$, chlorosulfonic acid $(0.19 \mathrm{~g}, 1.65 \mathrm{ml})$ was added dropwise at $0{ }^{\circ} \mathrm{C}$ over $2 \mathrm{~h}$. After the addition was complete the mixture was stirred for $2 \mathrm{~h}$ until $\mathrm{HCl}$ gas evolution was stopped. Then, the mixture was filtered and washed with ethanol $(30 \mathrm{ml})$ and dried at room temperature to obtain SASPSPE as a cream powder $(5.13 \mathrm{~g})$. The sulfur content of the samples by conventional elemental analysis was $15.51 \%$ [7].

\subsection{General procedure for the synthesis of 4,4'-(arylmethylene)bis(1 $H$-pyrazol-5-ols)}

A mixture of aromatic aldehyde (1 mmol), 5-methyl2-phenyl-2,4-dihydro-3H-pyrazol-3-one (2 mmol), and SASPSPE $(0.1 \mathrm{~g})$ in ethanol $(10 \mathrm{ml})$ were added to a flask and heated under reflux for an appropriate time. After completion of the reaction, as indicated by thin layer chromatography (TLC, silica gel SILG/UV 254 plates), the reaction mixture was washed with warm ethanol $(30 \mathrm{ml} \times 3)$. After cooling, the crude products were precipitated. The crude products were purified by recrystallization from ethanol (95\%). The recovered catalyst was washed with diethyl ether, dried, and reused in subsequent runs.

The infrared (IR) spectra were obtained on a Shimadzu IR-435. The ${ }^{1} \mathrm{H}$ NMR was carried out on a Bruker Avance (300 MHz or DRX $500 \mathrm{MHz}$ ) or Bruker Ultrashield (400 $\mathrm{MHz}$ ). Melting points were determined using a Melting Point SMP1 apparatus in open capillary tubes and are uncorrected. All the products were characterized by comparing their spectral (IR, ${ }^{1} \mathrm{H}$ NMR), TLC, and physical data with those reported in the literature [34-48].

4,4'-(Phenylmethylene)bis(3-methyl-1-phenyl-1H-pyra zol-5-ol) (3a). White cream solid; recrystallized from ethanol; mp $170-172{ }^{\circ} \mathrm{C}\left(171-172{ }^{\circ} \mathrm{C}\right.$ [45]). IR ( $\left.\mathrm{KBr}, \mathrm{cm}^{-1}\right)$ : 3400 (br), 3080 (w), 2900 (w), 1593 (s), 1494 (vs), 1410 (s), 1275 (s), 1020 (m), 730 (vs), 690 (s). ${ }^{1} \mathrm{H}$ NMR (300 MHz, DMSO- $\left.\mathrm{d}_{6}\right): \delta 2.32(6 \mathrm{H}, \mathrm{s}), 4.96(1 \mathrm{H}, \mathrm{s}), 7.17-7.27(7 \mathrm{H}, \mathrm{m})$, $7.44(4 \mathrm{H}, \mathrm{t}, J=7.72 \mathrm{~Hz}), 7.71(4 \mathrm{H}, \mathrm{d}, J=7.91 \mathrm{~Hz}), 13.96$ $\left(2 \mathrm{H}\right.$, br, OH). ${ }^{13} \mathrm{C}$ NMR (75 MHz, DMSO-d 6 ): $\delta$ 33.13, $120.52,125.55,125.88,127.17,128.12,128.90,142.22$, 146.29 .

4,4'-[(4-Methylphenyl)methylene]bis(3-methyl-1-phen yl-1H-pyrazol-5-ol) (3b). White solid; recrystallized from ethanol; mp 202-204 ${ }^{\circ} \mathrm{C}\left(203{ }^{\circ} \mathrm{C}\right.$ [40]). IR (KBr, cm $\left.{ }^{-1}\right)$ : 3440 (br), 3075 (w), 2830 (w), 1590 (s), 1495 (vs), 1408 (s), 1294 (s), 1020 (m), 800 (m), 744 (vs), 688 (s). ${ }^{1} \mathrm{H}$ NMR $\left(300 \mathrm{MHz}, \mathrm{DMSO}-\mathrm{d}_{6}\right): \delta 2.24(3 \mathrm{H}, \mathrm{s}), 2.30(6 \mathrm{H}, \mathrm{s}), 4.90$ $(1 \mathrm{H}, \mathrm{s}), 7.07(2 \mathrm{H}, \mathrm{d}, J=8.29 \mathrm{~Hz}), 7.13(2 \mathrm{H}, \mathrm{d}, J=8.10 \mathrm{~Hz})$, $7.24(2 \mathrm{H}, \mathrm{t}, J=7.35 \mathrm{~Hz}), 7.44(4 \mathrm{H}, \mathrm{t}, J=7.72 \mathrm{~Hz}), 7.70(4 \mathrm{H}$, d, $J=7.91 \mathrm{~Hz}), 13.93(2 \mathrm{H}, \mathrm{br}, \mathrm{OH}) .{ }^{13} \mathrm{C}$ NMR $(75 \mathrm{MHz}$, DMSO-d $\left.{ }_{6}\right): \delta 18.55,32.39,114.85,120.47,125.49,128.08$, $128.89,132.27,137.39,146.18,155.49$.

4,4'-[(4-isopropylphenyl)methylene]bis(3-methyl-1-ph enyl-1H-pyrazol-5-ol) (3c). White cream solid; recrystallized from ethanol; mp $132-134{ }^{\circ} \mathrm{C}$. IR $\left(\mathrm{KBr}, \mathrm{cm}^{-1}\right): 3435$ (br), 3080 (w), 2920 (w), 2830 (w), 1590 (s), 1495 (vs), 1408 (s), 1380 (m), 1365 (m), 1294 (s), 1020 (m), 779 (m), 744 (s), 688 (s). ${ }^{1} \mathrm{H}$ NMR (500 MHz, DMSO-d $\left.{ }_{6}\right): \delta 1.16(6 \mathrm{H}$, d, $J=6.9 \mathrm{~Hz}), 2.31(6 \mathrm{H}, \mathrm{s}), 2.79-2.85(1 \mathrm{H}, \mathrm{m}), 4.90(1 \mathrm{H}, \mathrm{s})$, $7.13(2 \mathrm{H}, \mathrm{d}, J=8.2 \mathrm{~Hz}), 7.18(2 \mathrm{H}, \mathrm{d}, J=8.1 \mathrm{~Hz}), 7.23(2 \mathrm{H}$, $\mathrm{t}, J=7.25 \mathrm{~Hz}), 7.43(4 \mathrm{H}, \mathrm{t}, J=7.3 \mathrm{~Hz}), 7.72(4 \mathrm{H}, \mathrm{d}, J=7.9$ $\mathrm{Hz}), 12.30$ (1H, br, OH), $14.03(1 \mathrm{H}, \mathrm{s}, \mathrm{OH}) .{ }^{13} \mathrm{C}$ NMR $(125$ 
MHz, DMSO- $\left.\mathrm{d}_{6}\right): \delta 12.51,24.78,33.70,33.84,121.39$, $126.40,126.93,127.93,129.77,140.57,146.72,147.14$. Anal. Calcd for $\mathrm{C}_{30} \mathrm{H}_{30} \mathrm{~N}_{4} \mathrm{O}_{2}(\%)$ : C, 75.29; H, 6.32; N, 11.71. Found: C, 75.12; H, 6.35; N, 11.54 .

4,4'-[(3,4,5-Trimethoxyphenyl)methylene]bis(3-methyl -1-phenyl-1H-pyrazol-5-ol) (3d). White cream solid; recrystallized from ethanol; $\mathrm{mp} 195-197{ }^{\circ} \mathrm{C}$. IR $\left(\mathrm{KBr}, \mathrm{cm}^{-1}\right)$ : 3447 (br), 3085 (w), 2885 (w), 1585 (s), 1490 (vs), 1443 (s), 1410 (s), 1318 (m), 1262 (m), 1122 (m), 898 (m), 850 (s), 790 (vs), 690 (s). ${ }^{1} \mathrm{H}$ NMR (500 MHz, DMSO-d 6 ): $\delta 2.33$ $(6 \mathrm{H}, \mathrm{s}), 3.62(3 \mathrm{H}, \mathrm{s}), 3.69(6 \mathrm{H}, \mathrm{s}), 4.85(1 \mathrm{H}, \mathrm{s}), 6.69(2 \mathrm{H}, \mathrm{s})$, $7.24(2 \mathrm{H}, \mathrm{t}, J=7.25 \mathrm{~Hz}), 7.43(4 \mathrm{H}, \mathrm{t}, J=7.81 \mathrm{~Hz}), 7.71(4 \mathrm{H}$, $\mathrm{d}, J=7.91 \mathrm{~Hz}), 14.29(1 \mathrm{H}, \mathrm{s}, \mathrm{OH}) .{ }^{13} \mathrm{C} \mathrm{NMR}(125 \mathrm{MHz}$, DMSO-d $\left._{6}\right): \delta 12.61,34.76,56.70,60.80,105.81,121.59$, $126.45,129.78,136.86,138.29,139.62,147.03,153.42$. Anal. Calcd for $\mathrm{C}_{30} \mathrm{H}_{30} \mathrm{~N}_{4} \mathrm{O}_{5}(\%)$ : C, 68.42; H, 5.74; N, 10.64 . Found: C, 68.19; H, 5.59; N, 10.38.

4,4'-[(2-Hydroxyphenyl)methylene]bis(3-methyl-1-phe nyl-1H-pyrazol-5-ol) (3e). White solid; recrystallized from ethanol; mp $227-229^{\circ} \mathrm{C}\left(230-231^{\circ} \mathrm{C}\right.$ [46]). IR ( $\left.\mathrm{KBr}, \mathrm{cm}^{-1}\right)$ : 3400 (br), 3060 (w), 2920 (w), 2830 (w), 1605 (s), 1575 (s), 1455 (vs), 1225 (m), 750 (s), 690 (s). ${ }^{1} \mathrm{H}$ NMR (500 MHz, DMSO-d $\left._{6}\right): \delta 2.28(6 \mathrm{H}, \mathrm{s}), 5.16(1 \mathrm{H}, \mathrm{s}), 6.70(1 \mathrm{H}, \mathrm{t}, J=7.5$ $\mathrm{Hz}), 6.74(1 \mathrm{H}, \mathrm{d}, J=7.5 \mathrm{~Hz}), 6.96(1 \mathrm{H}, \mathrm{t}, J=7.0 \mathrm{~Hz}), 7.22$ $(2 \mathrm{H}, \mathrm{t}, J=7.3 \mathrm{~Hz}), 7.42(4 \mathrm{H}, \mathrm{t}, J=7.9 \mathrm{~Hz}), 7.57(1 \mathrm{H}, \mathrm{d}, J=$ $7.2 \mathrm{~Hz}), 7.71(4 \mathrm{H}, \mathrm{d}, J=7.8 \mathrm{~Hz}) \cdot{ }^{13} \mathrm{C}$ NMR $(125 \mathrm{MHz}$, DMSO-d $\left._{6}\right): \delta 12.73,28.15,115.66,119.42,121.37,126.18$, $127.65,129.71,147.16,154.68$.

4,4'-[(2-Bromophenyl)methylene]bis(3-methyl-1-phen yl-1H-pyrazol-5-ol) (3f). White cream solid; recrystallized from ethanol; mp 198-200 ${ }^{\circ} \mathrm{C}$. IR ( $\left.\mathrm{KBr}, \mathrm{cm}^{-1}\right)$ : 3440 (br), 3060 (w), 2920 (w), 1605 (s), 1560 (s), 1495 (vs), 1395 (m), $1365(\mathrm{~m}), 1300(\mathrm{~m}), 830(\mathrm{~m}), 744$ (s), 690 (s). ${ }^{1} \mathrm{H}$ NMR $\left(500 \mathrm{MHz}, \mathrm{DMSO}-\mathrm{d}_{6}\right): \delta 2.31(6 \mathrm{H}, \mathrm{s}), 5.10(1 \mathrm{H}, \mathrm{s}), 7.13$ $(1 \mathrm{H}, \mathrm{t}, J=7.25 \mathrm{~Hz}), 7.23(2 \mathrm{H}, \mathrm{t}, J=7.0 \mathrm{~Hz}), 7.33(1 \mathrm{H}, \mathrm{t}, J$ $=7.3 \mathrm{~Hz}), 7.42(4 \mathrm{H}, \mathrm{t}, J=7.9 \mathrm{~Hz}), 7.56(1 \mathrm{H}, \mathrm{d}, J=7.8 \mathrm{~Hz})$, $7.71(4 \mathrm{H}, \mathrm{d}, J=7.9 \mathrm{~Hz}), 7.86(1 \mathrm{H}, \mathrm{m}), 12.68(1 \mathrm{H}, \mathrm{br}, \mathrm{OH})$, $13.92(1 \mathrm{H}, \mathrm{s}, \mathrm{OH}) .{ }^{13} \mathrm{C} \mathrm{NMR}\left(125 \mathrm{MHz}, \mathrm{DMSO}-\mathrm{d}_{6}\right): \delta$ $13.08,35.21,121.49,123.54,126.43,128.30,129.16$, 129.76, 131.44, 133.64, 138.21, 146.78. Anal. Calcd for $\mathrm{C}_{27} \mathrm{H}_{23} \mathrm{BrN}_{4} \mathrm{O}_{2}(\%)$ : C, 62.92; H, 4.50; Br, 15.50; N, 10.87. Found: C, 62.74; H, 4.54; N, 10.70 .

4,4'-[(4-Chlorophenyl)methylene]bis(3-methyl-1-phen yl-1H-pyrazol-5-ol) (3g). White solid; recrystallized from ethanol; mp $215-217{ }^{\circ} \mathrm{C}\left(210{ }^{\circ} \mathrm{C}\right.$ [40]). IR $\left(\mathrm{KBr}, \mathrm{cm}^{-1}\right)$ : 3430 (br), 3085 (w), 2920 (w), 1595 (s), 1490 (vs), 1410 (s), 1290 (m), 804 (m), 742 (s), 690 (s). ${ }^{1} \mathrm{H}$ NMR (300 MHz, DMSO-d $\left.{ }_{6}\right): \delta 2.30(6 \mathrm{H}, \mathrm{s}), 4.98(1 \mathrm{H}, \mathrm{s}) 7.22-7.28(4 \mathrm{H}, \mathrm{m})$, $7.35(2 \mathrm{H}, \mathrm{d}, J=8.48 \mathrm{~Hz}), 7.44(4 \mathrm{H}, \mathrm{t}, J=7.91 \mathrm{~Hz}), 7.71(4 \mathrm{H}$, $\mathrm{d}, J=7.91 \mathrm{~Hz}), 13.90(2 \mathrm{H}, \mathrm{br}, \mathrm{OH}) .{ }^{13} \mathrm{C}$ NMR $(75 \mathrm{MHz}$, DMSO-d ${ }_{6}$ ): $\delta 32.56,120.54,125.62,128.00,128.90,129.13$, $130.56,137.18,141.14,146.23$.
4,4'-[(2-Chlorophenyl)methylene]bis(3-methyl-1-phen yl-1H-pyrazol-5-ol) (3h). White solid; recrystallized from ethanol; mp 235-237 ${ }^{\circ} \mathrm{C}\left(236-237{ }^{\circ} \mathrm{C}\right.$ [45]). IR $\left(\mathrm{KBr}, \mathrm{cm}^{-1}\right)$ : 3450 (br), 3070 (w), 2910 (w), 1610 (m), 1555 (s), 1495 (vs), 1395 (m), 1360 (m), 1300 (s), 835 (m), 740 (s), 690 (s). ${ }^{1} \mathrm{H}$ NMR (400 MHz, DMSO-d 6 ): $\delta 2.29(6 \mathrm{H}, \mathrm{s}), 5.14(1 \mathrm{H}, \mathrm{s})$, $7.22-7.33(4 \mathrm{H}, \mathrm{m}), 7.40(1 \mathrm{H}, \mathrm{d}, J=7.82 \mathrm{~Hz}), 7.44(4 \mathrm{H}, \mathrm{t}, J$ $=7.57 \mathrm{~Hz}), 7.70(4 \mathrm{H}, \mathrm{d}, J=7.57 \mathrm{~Hz}), 7.80(1 \mathrm{H}, \mathrm{d}, J=7.06$ $\mathrm{Hz}), 13.92$ (2H, br, OH). ${ }^{13} \mathrm{C}$ NMR (100 MHz, DMSO-d 6 ): $\delta$ $32.41,120.67,123.62,126.92,128.05,128.93,129.45$, 130.32, 135.94, 137.36, 140.60, 141.18 .

4,4'-[(4-Flurophenyl)methylene]bis(3-methyl-1-phenyl -1H-pyrazol-5-ol) (3i). White solid; recrystallized from ethanol; mp $181-183{ }^{\circ} \mathrm{C}\left(182{ }^{\circ} \mathrm{C}\right.$ [40]). IR ( $\left.\mathrm{KBr}, \mathrm{cm}^{-1}\right)$ : 3445 (br), 3080 (w), 2882 (w), 1590 (s), 1495 (vs), 1401 (m), 1380 (s), 1308 (m), 1121 (m), 902 (s), 842 (m), 788 (s), 690 (s). ${ }^{1} \mathrm{H}$ NMR (500 MHz, DMSO-d $\left.{ }_{6}\right): \delta 2.31(6 \mathrm{H}, \mathrm{s})$, $4.95(1 \mathrm{H}, \mathrm{s}), 7.09(2 \mathrm{H}, \mathrm{t}, J=8.75 \mathrm{~Hz}), 7.22-7.28(4 \mathrm{H}, \mathrm{m})$, $7.43(4 \mathrm{H}, \mathrm{t}, J=7.65 \mathrm{~Hz}), 7.70(4 \mathrm{H}, \mathrm{d}, J=7.90 \mathrm{~Hz}), 12.48$ $\left(1 \mathrm{H}\right.$, br, OH), $13.91(1 \mathrm{H}, \mathrm{s}, \mathrm{OH}) .{ }^{13} \mathrm{C}$ NMR $(125 \mathrm{MHz}$, DMSO-d $\left.{ }_{6}\right): \delta 12.43,33.32,115.51,115.68,121.42,126.45$, $129.74,129.78,129.85,129.92,139.07,147.11,162.50$.

4,4'-[(4-Nitrophenyl)methylene]bis(3-methyl-1-phenyl -1H-pyrazol-5-ol) (3j). Yellow solid; recrystallized from ethanol; mp 225-227 ${ }^{\circ} \mathrm{C}\left(224-226^{\circ} \mathrm{C}\right.$ [45]). IR ( $\left.\mathrm{KBr}, \mathrm{cm}^{-1}\right)$ : 3440 (br), 3090 (w), 2920 (w), 1595 (s), 1495 (vs), 1410 (s), 1340 (m), 744 (s), 689 (s). ${ }^{1} \mathrm{H}$ NMR (400 MHz, DMSO-d 6 ): $\delta 2.28(6 \mathrm{H}, \mathrm{s}), 5.06(1 \mathrm{H}, \mathrm{s}), 7.18(2 \mathrm{H}, \mathrm{t}, J=7.06 \mathrm{~Hz}), 7.38$ $(4 \mathrm{H}, \mathrm{t}, J=7.31 \mathrm{~Hz}), 7.45(2 \mathrm{H}, \mathrm{d}, J=8.32 \mathrm{~Hz}), 7.64(4 \mathrm{H}, \mathrm{d}$, $J=7.82 \mathrm{~Hz}), 8.10(2 \mathrm{H}, \mathrm{d}, J=8.58 \mathrm{~Hz}), 13.81(2 \mathrm{H}, \mathrm{br}, \mathrm{OH})$. ${ }^{13} \mathrm{C}$ NMR (100MHz, DMSO-d 6 ): $\delta 34.45,121.91,124.65$, $127.03,129.92,130.25,147.20,147.58,151.63$.

4,4'-[(3-Nitrophenyl)methylene]bis(3-methyl-1-phenyl -1H-pyrazol-5-ol) (3k). Pale yellow solid; recrystallized from ethanol; $\mathrm{mp} 151-153{ }_{0} \mathrm{C}\left(149-150{ }^{\circ} \mathrm{C}\right.$ [45]). IR (KBr, $\mathrm{cm}^{-1}$ ): 3420 (br), 3085 (w), 2910 (w), 1595 (s), 1495 (vs), 1340 (s), 758 (m), 735 (s), 692 (s), 598 (m). ${ }^{1} \mathrm{H}$ NMR (400 MHz, DMSO-d $\left.{ }_{6}\right): \delta 2.35(6 \mathrm{H}, \mathrm{s}), 5.14(1 \mathrm{H}, \mathrm{s}), 7.26(2 \mathrm{H}, \mathrm{t}, J$ $=7.31 \mathrm{~Hz}), 7.45(4 \mathrm{H}, \mathrm{t}, J=7.57 \mathrm{~Hz}), 7.60(1 \mathrm{H}, \mathrm{t}, J=8.32$ $\mathrm{Hz}), 7.68-7.74$ (5H, m), 8.06-8.10 (2H, m), 13.91 (2H, br, $\mathrm{OH}) .{ }^{13} \mathrm{C}$ NMR $\left(100 \mathrm{MHz}, \mathrm{DMSO}-\mathrm{d}_{6}\right): \delta 32.80,120.63$, $121.21,121.70,125.78,125.81,128.98,129.71,134.34$, $137.39,144.56,146.30,147.72$.

4,4'-[(4-Cyanophenyl)methylene]bis(3-methyl-1-pheny l-1H-pyrazol-5-ol) (3I). Yellow solid; recrystallized from ethanol; mp $210-212^{\circ} \mathrm{C}\left(210-212^{\circ} \mathrm{C}\right.$ [17]). IR ( $\left.\mathrm{KBr}, \mathrm{cm}^{-1}\right)$ : 3420 (br), 3090 (w), 2921 (w), 2230 (m), 1595 (s), 1495 (vs), 1410 (s), 1290 (m), 810 (m), 750 (s), 690 (s). ${ }^{1} \mathrm{H}$ NMR (400 MHz, DMSO-d $\left.\mathrm{d}_{6}\right): \delta 2.33(6 \mathrm{H}, \mathrm{s}), 5.07(1 \mathrm{H}, \mathrm{s}), 7.25$ $(2 \mathrm{H}, \mathrm{t}, J=7.31 \mathrm{~Hz}), 7.42-7.46(6 \mathrm{H}, \mathrm{m}), 7.40(4 \mathrm{H}, \mathrm{d}, J=$ $7.82 \mathrm{~Hz}), 7.76(2 \mathrm{H}, \mathrm{d}, J=8.32 \mathrm{~Hz}), 13.89(2 \mathrm{H}, \mathrm{br}, \mathrm{OH}) .{ }^{13} \mathrm{C}$ NMR (100 MHz, DMSO-d 6 ): $\delta 33.23,119.00,120.61$, 
$125.57,128.38,128.94,133.36,142.59,148.15$.

\section{4,4'-[(2-Naphthyl)methylene]bis(3-methyl-1-phenyl-1}

H-pyrazol-5-ol) (3m). White solid; recrystallized from ethanol; $\mathrm{mp} 206-208{ }^{\circ} \mathrm{C}$. IR $\left(\mathrm{KBr}, \mathrm{cm}^{-1}\right)$ : 3410 (br), 3030 (w), 1590 (s), 1490 (vs), 1392 (s), 1360 (m), 1280 (m), 1020 (m), 810 (m), 780 (m), 740 (s), 690 (s); ${ }^{1} \mathrm{H}$ NMR (500 MHz, DMSO-d $\left._{6}\right): \delta 2.36(6 \mathrm{H}, \mathrm{s}), 5.14(1 \mathrm{H}, \mathrm{s}), 7.24(2 \mathrm{H}, \mathrm{t}, J=6.9$ $\mathrm{Hz}), 7.41-7.45(7 \mathrm{H}, \mathrm{m}), 7.71-7.73(5 \mathrm{H}, \mathrm{m}), 7.81-7.85(3 \mathrm{H}$, $\mathrm{m}), 12.41(1 \mathrm{H}, \mathrm{br}, \mathrm{OH}), 13.93(1 \mathrm{H}, \mathrm{s}, \mathrm{OH}) ;{ }^{13} \mathrm{C}$ NMR $(125$ MHz, DMSO-d $)_{6}: \delta 12.50,34.22,121.46,125.80,126.30$, $126.83,127.36,128.16,128.52,128.59,129.79,132.54$, 133.73, 140.55, 147.19. Anal. Calcd for $\mathrm{C}_{31} \mathrm{H}_{26} \mathrm{~N}_{4} \mathrm{O}_{2}(\%)$ : $\mathrm{C}$, 76.52; H, 5.39; N, 11.51; Found: C, 76.35; H, 5.30; N, 11.46 .

4,4'-[(3-Pyridyl)methylene]bis(3-methyl-1-phenyl-1Hpyrazol-5-ol) (3n). White cream solid; recrystallized from ethanol; mp 238-240 ${ }^{\circ} \mathrm{C}$. IR ( $\mathrm{KBr}, \mathrm{cm}^{-1}$ ): 3410 (br), 3050 (w), 2900 (w), 1595 (s), 1490 (vs), 1410 (s), 1345 (m), 1280 (m), 1020 (m), 850 (m), 790 (m), 745 (s), 695 (s); ${ }^{1} \mathrm{H}$ NMR $\left(500 \mathrm{MHz}, \mathrm{DMSO}-\mathrm{d}_{6}\right): \delta 2.34(6 \mathrm{H}, \mathrm{s}), 5.05(1 \mathrm{H}, \mathrm{s}), 7.23$ $(2 \mathrm{H}, \mathrm{t}, J=7.1 \mathrm{~Hz}), 7.34(1 \mathrm{H}, \mathrm{t}, J=6.0 \mathrm{~Hz}), 7.43(4 \mathrm{H}, \mathrm{t}, J=$ $7.5 \mathrm{~Hz}), 7.71-7.73(5 \mathrm{H}, \mathrm{m}), 8.41(1 \mathrm{H}, \mathrm{d}, J=3.6 \mathrm{~Hz}), 8.51$ $(1 \mathrm{H}, \mathrm{s}), 12.10(1 \mathrm{H}, \mathrm{br}, \mathrm{OH}), 14.12(1 \mathrm{H}, \mathrm{br}, \mathrm{OH}) ;{ }^{13} \mathrm{C} \mathrm{NMR}$ (125 MHz, DMSO-d ${ }_{6}$ ): $\delta 12.54,31.96,104.68,121.45$, $124.22,126.44,129.76,136.16,138.25,138.91,147.04$, 147.61, 149.32. Anal. Calcd for $\mathrm{C}_{26} \mathrm{H}_{23} \mathrm{~N}_{5} \mathrm{O}_{2}$ (\%): C, 71.38; $\mathrm{H}, 5.30 ; \mathrm{N}, 16.01$; Found C, 71.20; H, 5.25; N, 15.87 .

4,4'-[(2-Thienyl)methylene]bis(3-methyl-1-phenyl-1Hpyrazol-5-ol) (3o). White cream solid; recrystallized from ethanol; $\mathrm{mp} 181-183{ }^{\circ} \mathrm{C}\left(181-183^{\circ} \mathrm{C}\right.$ [17]). IR ( $\left.\mathrm{KBr}, \mathrm{cm}^{-1}\right)$ : 3420 (br), 3080 (w), 2920 (w), 1595 (s), 1490 (vs), 1410 (s), 1284 (s), 779 (vs), 690 (s). ${ }^{1} \mathrm{H}$ NMR (400 MHz, DMSO-d 6 ): $\delta 2.32(6 \mathrm{H}, \mathrm{s}), 5.13(1 \mathrm{H}, \mathrm{s}), 6.75-6.77(1 \mathrm{H}, \mathrm{m}), 6.90-6.92$ $(1 \mathrm{H}, \mathrm{m}), 7.24-7.30(3 \mathrm{H}, \mathrm{m}), 7.45(4 \mathrm{H}, \mathrm{t}, J=7.82 \mathrm{~Hz}), 7.71$ $(4 \mathrm{H}, \mathrm{d}, J=7.82 \mathrm{~Hz}) 14.01(2 \mathrm{H}, \mathrm{br}, \mathrm{OH}) .{ }^{13} \mathrm{C} \mathrm{NMR}$ $\left(100 \mathrm{MHz}, \mathrm{DMSO}-\mathrm{d}_{6}\right): \delta 29.43,120.58,124.05,124.15$, $126.75,128.94,132.99,134.13,147.73$.

4,4'-[(2-Furyl)methylene]bis(3-methyl-1-phenyl-1H-py razol-5-ol) (3p). White cream solid; recrystallized from ethanol; mp 189-191 ${ }^{\circ} \mathrm{C}\left(181-183{ }^{\circ} \mathrm{C}\right.$ [47]). IR ( $\left.\mathrm{KBr}, \mathrm{cm}^{-1}\right)$ : 3420 (br), 3080 (w), 2920 (w), 1595 (s), 1490 (vs), 1410 (s), 1284 (s), 779 (vs), 690 (s). ${ }^{1} \mathrm{H}$ NMR (400 MHz, DMSO-d 6 ): $\delta 2.31(6 \mathrm{H}, \mathrm{s}), 4.99(1 \mathrm{H}, \mathrm{s}), 6.12-6.14(1 \mathrm{H}, \mathrm{m}), 6.34-6.36$ $(1 \mathrm{H}, \mathrm{m}), 7.25(2 \mathrm{H}, \mathrm{t}, J=6.0 \mathrm{~Hz}), 7.43-7.51(5 \mathrm{H}, \mathrm{m}), 7.71$ $(4 \mathrm{H}, \mathrm{d}, J=8.0 \mathrm{~Hz}), 12.46(1 \mathrm{H}, \mathrm{br}, \mathrm{OH}), 13.85(1 \mathrm{H}, \mathrm{s}, \mathrm{OH})$. ${ }^{13} \mathrm{C}$ NMR $\left(100 \mathrm{MHz}, \mathrm{DMSO}-\mathrm{d}_{6}\right): \delta 28.22,106.11,109.88$, $110.34,120.43,120.55,125.60,128.91,131.92,141.54$.

1,3-Diphenylene-4,4'-(methylene)bis(3-methyl-1-phen yl-1H-pyrazol-5-ol) (4a). White cream solid; recrystallized from ethanol; mp $191-192{ }^{\circ} \mathrm{C}\left(190-194{ }^{\circ} \mathrm{C}\right.$ [48]). IR (KBr, $\mathrm{cm}^{-1}$ ): 3405 (br), 3015 (w), 1599 (s), 1402 (vs), 1279 (vs), 1025 (m), 788 (m), 753 (vs), 683 (vs). ${ }^{1} \mathrm{H}$ NMP (400 MHz,
DMSO-d $\left._{6}\right): \delta 2.25(12 \mathrm{H}, \mathrm{s}), 4.86(2 \mathrm{H}, \mathrm{s}), 7.07-7.67(24 \mathrm{H}$, $\mathrm{m}), 12.45(2 \mathrm{H}, \mathrm{br}, \mathrm{OH}) 14.08(2 \mathrm{H}, \mathrm{s}, \mathrm{OH}) .{ }^{13} \mathrm{C}$ NMP $(100$ $125 \mathrm{MHz}$, DMSO-d $\left.{ }_{6}\right): \delta 18.54,33.34,120.70,125.87$, $126.68,128.82,134.52,142.40,149.89,154.26$.

1,4-Diphenylene-4,4'-(methylene)bis(3-methyl-1-phen yl-1H-pyrazol-5-ol) (4b). White cream solid; recrystallized from ethanol; $\mathrm{mp} 214-216{ }^{\circ} \mathrm{C}\left(218-220{ }^{\circ} \mathrm{C}\right.$ [48]). IR (KBr, $\mathrm{cm}^{-1}$ ): 3410 (br), 3020 (w), 1590 (s), 1490 (vs), 1410 (s), 1350 (s), 1290 (s), 1120 (s), 1020 (m), 850 (m), 745 (vs), 690 (s). ${ }^{1} \mathrm{H}$ NMR (500 MHz, DMSO-d 6 ): $\delta 2.29(12 \mathrm{H}, \mathrm{s})$, $5.05(2 \mathrm{H}, \mathrm{s}), 7.17(4 \mathrm{H}, \mathrm{s}), 7.22(4 \mathrm{H}, \mathrm{t}, J=7.1 \mathrm{~Hz}), 7.41(8 \mathrm{H}$, $\mathrm{t}, J=7.8 \mathrm{~Hz}), 7.69(8 \mathrm{H}, \mathrm{d}, J=7.9 \mathrm{~Hz}), 12.41(2 \mathrm{H}, \mathrm{br}, \mathrm{OH})$ $14.11(2 \mathrm{H}, \mathrm{s}, \mathrm{OH}) .{ }^{13} \mathrm{C}$ NMR $\left(125 \mathrm{MHz}, \mathrm{DMSO}-\mathrm{d}_{6}\right): \delta 12.51$, $33.69,121.50,127.84,129.72,140.93,147.07,155.01$. Anal. Calcd for $\mathrm{C}_{48} \mathrm{H}_{42} \mathrm{~N}_{8} \mathrm{O}_{4}(\%)$ : C, 72.52; H, 5.32; N, 14.09. Found: C, 72.35; H, 5.29; N, 13.85 .

\section{Results and discussion}

To study the effect of catalyst loading on the condensation reaction of aromatic aldehydes with 3-methyl-1-phenyl5-pyrazolone the reaction between benzaldehyde and 5-methyl-2-phenyl-2,4-dihydro-3H-pyrazol-3-one was chosen as the model reaction (Table 1). The results show clearly that SASPSPE is an effective catalyst for this condensation and in the absence of SASPSPE the condensation reaction gave a very low yield after $24 \mathrm{~h}$. The optimal amount of SASPSPE was $0.1 \mathrm{~g}$ (3.4 mol\%) per $1 \mathrm{mmol}$ of aldehyde in refluxing ethanol. Although the lower catalyst loading of $0.05 \mathrm{~g}$ of SASPSPE accomplished this condensation, $0.1 \mathrm{~g}$ SASPSPE per $1 \mathrm{mmol}$ of aldehyde was optimum in terms of the reaction time and the isolated yield. Also, the model

Table 1 Condensation reaction of benzaldehyde with 5-methyl-2phenyl-2,4-dihydro-3H-pyrazol-3-one in the presence of different amounts of catalysts

\begin{tabular}{ccccc}
\hline Entry & Catalyst & Catalyst loading & Time (h) & Yield $^{\mathrm{a}}(\%)$ \\
\hline 1 & no catalyst & - & 24 & $<10$ \\
2 & SASPSPE & $0.05 \mathrm{~g}(0.017 \mathrm{mmol})$ & 6.5 & 70 \\
3 & SASPSPE & $0.07 \mathrm{~g}(0.023 \mathrm{mmol})$ & 5.0 & 78 \\
4 & SASPSPE & $0.1 \mathrm{~g}(0.034 \mathrm{mmol})$ & 3.0 & 90 \\
5 & $\mathrm{SASPSPE}$ & $0.15 \mathrm{~g}(0.051 \mathrm{mmol})$ & 3.0 & 89 \\
6 & $\mathrm{SBNPSA}$ & $0.2 \mathrm{~g}(0.068 \mathrm{mmol})$ & 4.0 & 90 \\
7 & $\mathrm{H}_{2} \mathrm{SO}_{4}$ & $0.2 \mathrm{mmol}$ & 4.0 & $44^{\mathrm{c}}$ \\
8 & $\mathrm{H}_{2} \mathrm{SO}_{4}$ & $1.0 \mathrm{mmol}$ & 3.0 & $44^{\mathrm{c}}$ \\
9 & $\mathrm{H}_{2} \mathrm{SO}_{4}$ & $4.0 \mathrm{mmol}$ & 1.2 & $45^{\mathrm{c}}$ \\
10 & $\mathrm{H}_{2} \mathrm{SO}_{4}$ & $10.0 \mathrm{mmol}$ & 0.7 & $47^{\mathrm{c}}$ \\
11 & $\mathrm{SASPSPE}$ & $0.1 \mathrm{~g}(0.034 \mathrm{mmol})$ & 15.0 & $55^{\mathrm{d}}$ \\
\hline
\end{tabular}

Reaction conditions: benzaldehyde $1 \mathrm{mmol}$, 5-methyl-2-phenyl-2,4dihydro-3H-pyrazol-3-one $2 \mathrm{mmol}$, EtOH $10 \mathrm{ml}$, reflux conditions. ${ }^{a}$ Isolated yield. ${ }^{b}$ Silica-bonded $N$-propyl-sulfamic acid (SBNPSA) [8]. ${ }^{\mathrm{c}}$ The same yield was obtained for the by-product. ${ }^{\mathrm{d}} \mathrm{GC}$ yield of the conversion at room temperature. 
reaction was examined using optimum amounts and at room temperature, and we obtained a GC yield of $55 \%$ for the corresponding product (Table 1, entry 11). In addition, the result of this condensation in the presence of commercially available sulfuric acid is shown in Table 1.

Therefore, we employed optimized conditions for the condensation reaction of various aryl aldehydes with 3-methyl-1-phenyl-5-pyrazolone to give the corresponding 4,4'-(arylmethylene) bis (3-methyl-1-phenyl-1H-pyrazol-5ols) (Scheme 2).

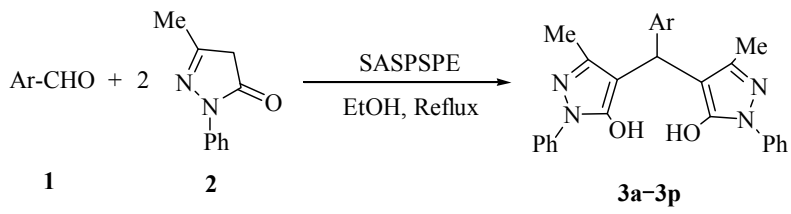

Scheme 2. The condensation of aromatic aldehydes with 3-methyl1-phenyl-5-pyrazolone upon catalysis by SASPSPE.

As shown in Table 2, both the aromatic and heteroaromatic aldehydes react with 3-methyl-1-phenyl-5-pyrazolone to afford 4,4'-(arylmethylene)-bis-(3-methyl-1-phenyl-1 $H$ pyrazol-5-ols) in excellent yields. On the other hand, benzaldehydes with electron-donating or electron-withdrawing groups, i.e. methyl, iso-propyl, and 3,4,5-trimethoxybenzaldehyde (Table 2, entries 2-4) or 4-nitro, 3-nitro, and 4-cyano-benzaldehyde (Table 2, entries 10-12) condense into the corresponding 4,4'-(arylmethylene)-bis-(3methyl-1-phenyl-1H-pyrazol-5-ols) $\mathbf{3 b} \mathbf{- 3 d}$ and $\mathbf{3 j} \mathbf{j}-\mathbf{3 l}$ in high yields. The acid sensitive substrates 3 -pyridine carbalde- hyde, thiophene-2-carbaldehyde, and furfural (Table 2, entries 14-16) were converted into the corresponding products 3n, 3o, and 3p in $84 \%, 74 \%$, and $75 \%$ yields, respectively. 2-Naphthyl carbaldehyde was converted into the corresponding product $\mathbf{3 m}$ in $88 \%$ yield (Table 2, entry 13 ). Cinnamyl aldehyde was reacted with 3-methyl-1-phenyl5-pyrazolone under the optimized conditions but gave a mixture of products.

The practical synthetic efficiency of this reaction was highlighted by the reaction of terephthaldehyde and isophthaldehyde with 3-methyl-1-phenyl-5-pyrazolone to give structurally complex pyrazol-5-ol derivatives (4a and 4b, Scheme 3).

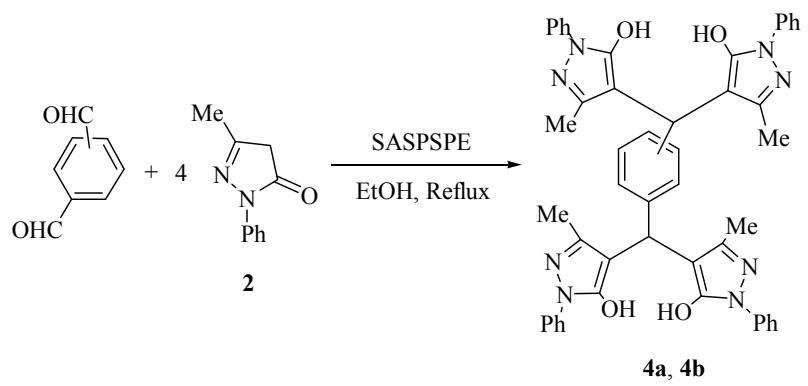

Scheme 3. The condensation of terephthaldehyde with 3-methyl-1phenyl-5-pyrazolone catalyzed by SASPSPE.

The possibility of recycling the catalyst SASPSPE was examined using the reaction of benzaldehyde and 5-methyl-2-phenyl-2,4-dihydro-3H-pyrazol-3-one under the optimized conditions. Upon completion, the reaction mix-

Table 2 Preparation of 4,4'-(arylmethylene)-bis-(3-methyl-1-phenyl-1H-pyrazol-5-ols) derivatives catalyzed by SASPSPE in ethanol under reflux conditions

\begin{tabular}{|c|c|c|c|c|c|}
\hline Entry & $\operatorname{Ar}(1)$ & Product & Time $(\mathrm{h})$ & Yield $^{\mathrm{a}}(\%)$ & $\mathrm{mp}\left({ }^{\circ} \mathrm{C}\right)$ \\
\hline 1 & $\mathrm{C}_{6} \mathrm{H}_{5^{-}}$ & $3 \mathbf{a}$ & 3.0 & 90 & $170-172(171-172[45])$ \\
\hline 2 & 4-Me- $\mathrm{C}_{6} \mathrm{H}_{4-}$ & $\mathbf{3 b}$ & 2.3 & 89 & $202-204(203[40])$ \\
\hline 3 & 4-iso-Pr- $\mathrm{C}_{6} \mathrm{H}_{4-}$ & $3 \mathbf{c}$ & 3.5 & 88 & $132-134$ \\
\hline 4 & $3,4,5-(\mathrm{MeO})_{3}-\mathrm{C}_{6} \mathrm{H}_{2}-$ & 3d & 3.3 & 89 & $195-197$ \\
\hline 5 & $2-\mathrm{HO}-\mathrm{C}_{6} \mathrm{H}_{4}^{-}$ & $3 e$ & 3.5 & 83 & $227-229(230-231[46])$ \\
\hline 6 & $2-\mathrm{Br}-\mathrm{C}_{6} \mathrm{H}_{4-}$ & $3 f$ & 2.6 & 78 & $198-200$ \\
\hline 7 & $4-\mathrm{Cl}-\mathrm{C}_{6} \mathrm{H}_{4}-$ & $3 g$ & 2.2 & 85 & $215-217(210[40])$ \\
\hline 8 & 2-Cl- $\mathrm{C}_{6} \mathrm{H}_{4}-$ & $3 \mathbf{h}$ & 2.4 & 77 & $235-237(236-237[45])$ \\
\hline 9 & $4-\mathrm{F}-\mathrm{C}_{6} \mathrm{H}_{4-}^{-}$ & $3 \mathbf{i}$ & 2.5 & 86 & $181-183(182[40])$ \\
\hline 10 & $4-\mathrm{O}_{2} \mathrm{~N}-\mathrm{C}_{6} \mathrm{H}_{4}-$ & $3 \mathbf{j}$ & 2.0 & 88 & $225-227(224-226[45])$ \\
\hline 11 & $3-\mathrm{O}_{2} \mathrm{~N}-\mathrm{C}_{6} \mathrm{H}_{4}-$ & $3 \mathbf{k}$ & 2.3 & 87 & $151-153(149-150[45])$ \\
\hline 12 & $4-(\mathrm{CN})-\mathrm{C}_{6} \mathrm{H}_{4-}^{-}$ & 31 & 2.1 & 89 & $210-212(210-212[17])$ \\
\hline 13 & $2-\mathrm{C}_{10} \mathrm{H}_{7^{-}}$ & $3 \mathrm{~m}$ & 3.0 & 88 & $206-208$ \\
\hline 14 & 3-pyridyl- & $3 n$ & 3.0 & 84 & $238-240$ \\
\hline 15 & 2-thienyl- & 30 & 4.0 & 74 & $181-183(181-183[17])$ \\
\hline 16 & 2-furyl- & $3 \mathbf{p}$ & 4.0 & 75 & 189-191 (189-190 [47]) \\
\hline 17 & $3-\mathrm{OHC}-\mathrm{C}_{6} \mathrm{H}_{4}-$ & $4 a$ & 4.0 & 82 & $191-192(190-194$ [48]) \\
\hline 18 & $4-\mathrm{OHC}-\mathrm{C}_{6} \mathrm{H}_{4-}$ & $4 b$ & 4.0 & 86 & $214-216(218-220[48])$ \\
\hline
\end{tabular}

Reaction conditions: aromatic aldehyde $1 \mathrm{mmol}$, 5-methyl-2-phenyl-2,4-dihydro-3H-pyrazol-3-one 2 mmol, catalyst SASPSPE 0.1 g, EtOH 10 ml, reflux conditions. ${ }^{a}$ Isolated yield. 
ture was washed with warm ethanol $(30 \mathrm{ml} \times 3)$. The recovered catalyst was washed with diethyl ether, dried, and reused for subsequent runs. The recycled catalyst was reused eleven times without any additional treatment. No observation of any appreciable loss in the catalytic activity of SASPSPE was observed (Fig. 1).

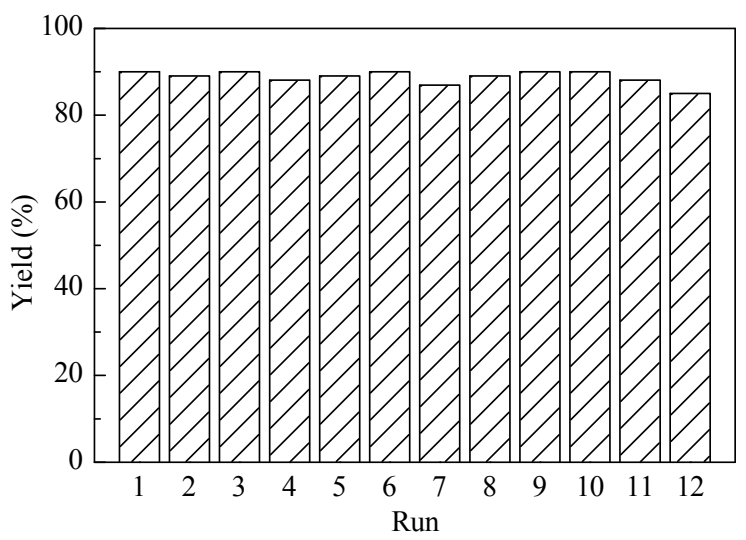

Fig. 1. Recyclability of SASPSPE as a catalyst in the condensation reaction between benzaldehyde $(1 \mathrm{mmol})$ and 5-methyl-2-phenyl-2,4dihydro-3 $H$-pyrazol-3-one $(2 \mathrm{mmol})$ in the presence of $0.1 \mathrm{~g}$ of SASPSPE in refluxing ethanol. Reaction time $3 \mathrm{~h}$.

\section{Conclusions}

We prepared new 4,4'-(arylmethylene)-bis-(3-methyl1-phenyl- $1 H$-pyrazol-5-ols) by a tandem condensation reaction between aromatic aldehydes and two equivalents of 5-methyl-2-phenyl-2,4-dihydro-3H-pyrazol-3-one in the presence of sulfuric acid ([3-(3-silicapropyl)sulfanyl]propyl) ester in refluxing ethanol.

\section{Acknowledgments}

We are grateful to the Islamic Azad University Research Council for partial support of this work.

\section{References}

1 Choudhary D, Paul S, Gupta R, Clark J H. Green Chem, 2006, 8: 479

2 Li Z, Ma X L, Liu J, Feng X, Tian G Q, Zhu A G. J Mol Catal A, 2007, 272: 132

3 Karimi B, Ghoreishi-Nezhad M. J Mol Catal A, 2007, 277: 262

4 Zareyee D, Karimi B. Tetrahedron Lett, 2007, 48: 1277

5 Melero J A, Grieken R V, Morales G. Chem Rev, 2006, 106: 3790

6 Shimizu K, Hayashi E, Hatamachi T, Kodama T, Higuch T, Satsuma A, Kitayama Y. J Catal, 2005, 231: 131

7 Niknam K, Saberi D. Appl Catal A, 2009, 366: 220

8 Niknam K, Saberi D. Tetrahedron Lett, 2009, 50: 5210
9 Niknam K, Saberi D, Nouri Sefat M. Tetrahedron Lett, 2009, 50: 4058

10 Niknam K, Zolfigol M A, Khorramabadi-Zad A, Zare R, Shayegh M. Catal Commun, 2006, 7: 494

11 Niknam K, Karami B, Zolfigol M A. Catal Commun, 2007, 8: 1427

12 Niknam K, Saberi D, Molaee H, Zolfigol M A. Can J Chem, 2010, 88: 164

13 Niknam K, Zolfigol M A, Chehardoli G, Dehghanian M. Chin J Catal, 2008, 29: 901

14 Niknam K, Zolfigol M A, Sadabadi T. J Iran Chem Soc, 2007, 4: 199

15 Niknam K, Zolfigol M A, Hossieninejad Z, Daneshvar N. Chin J Catal, 2007, 28: 591

16 Niknam K, Zolfigol M A, Sadabadi T, Nejati A. J Iran Chem Soc, 2006, 3: 318

17 Niknam K, Saberi D, Sadegheyan M, Deris A. Tetrahedron Lett, 2010, 51: 692

18 Niknam K, Saberi D, Baghernejad M. Phosphorus Sulfur Silicon Relat Elem, 2010, 185: 875

19 Niknam K, Panahi F, Saberi D, Mohagheghnejad M. J Heterocycl Chem, 2010, 47: 292

20 Niknam K, Saberi D, Mohagheghnejad M. Molecules, 2009, 14: 1915

21 Niknam K, Saberi D, Baghernejad B. Chin Chem Lett, 2009, 20: 1444

22 McDonald E, Jones K, Brough P A, Drysdale M J, Workman P. Curr Top Med Chem, 2006, 6: 1193

23 Elguero J. In: Katritzky A R, Rees C W, Scriven E F V eds. Comprehensive Heterocyclic Chemistry II. Vol. 5. Oxford: Pergamon Press, 1996

24 Elguero J, Goya P, Jagerovic N, Silva A M S. Targets Heterocycl Syst, 2002, 6: 52

25 Sugiura S, Ohno S, Ohtani O, Izumi K, Kitamikado T, Asai H, Kato K. J Med Chem, 1977, 20: 80

26 Behr L C, Fusco R, Jarboe C H. In: Weissberger A Ed. The Chemistry of Heterocyclic Compounds, Pyrazoles, Pyrazolines, Pyrazolidines, Indazoles, and Condensed Rings. New York: Interscience, 1967

27 Rosiere C E, Grossman M I. Science, 1951, 113: 651

28 Bailey D M, Hansen P E, Hlavac A G, Baizman E R, Pearl J, Defelice A F, Feigenson M E. J Med Chem, 1985, 28: 256

29 Mahajan R N, Havaldar F H, Fernandes P S. J Indian Chem Soc, 1991, 68: 245

30 Chauhan P M S, Singh S, Chatterjee R K. Indian J Chem, Sect $B, 1993$, 32B: 858

31 Singh D, Singh D. J Indian Chem Soc, 1991, 68: 165

32 Londershausen M. Pestic Sci, 1996, 48: 269

33 Lubs H A Ed. The Chemistry of Synthetic Dyes and Pigments. Washington D C: American Chemical Society, 1970

34 Uzoukwu A B, Al-Juaid S S, Hitchcock P B, Smith J D. Polyhedron, 1993, 12: 2719

35 Maurya R C, Verma R. Indian J Chem, Sect A, 1997, 36A: 596

36 Garnovskii A D, Uraev A I, Minkin V I. Arkivoc, 2004, Part 3: 29 
37 Sridhar R, Perumal P T, Etti S, Shanmugam G, Ponnusamy M N, Prabavathy V R, Mathivanan N. Bioorg Med Chem Lett, 2004, 14: 6035

38 Sivaprasad G, Perumal P T, Prabavathy V R, Mathivanan N. Bioorg Med Chem Lett, 2006, 16: 6302

39 Hamama W S. Synth Commun, 2001, 31: 1335

40 Li X L, Wang Y M, Tian B, Matsuura T, Meng J B. J Heterocycl Chem, 1998, 35: 129

41 Singh D, Singh D. J Chem Eng Data, 1984, 29: 355

42 Mitra A S, Rout M K J Indian Chem Soc, 1961, 38: 893

43 Pavlov P T, Goleneva A F, Lesnov A E, Prokhorova T S.
Pharm Chem J(Engl Trans), 1998, 32: 370

44 Buzykin B I, Lonshchakova T I. Bull Acad Sci USSR, Div Chem Sci (Engl Trans), 1971: 2224

45 Wang W, Wang S X, Qin X Y, Li J T. Synth Commun, 2005, 35: 1263

46 Elinson M N, Dorofeev A S, Nasybullin R F, Nikishin G I. Synthesis, 2008: 1933

47 Sujatha K, Shanthi G, Selvam N P, Manoharan S, Perumal P T, Rajendran M. Bioorg Med Chem Lett, 2009, 19: 4501

48 Reiner K, Richter R, Hauptmann S, Becher J, Hennig L. Tetrahedron, 1995, 51: 13291 